\title{
TEICHMÜLLER-TYPE ANNIHILATORS OF $L^{1}$-ANALYTIC FUNCTIONS
}

\author{
EDGAR REICH AND HAROLD S. SHAPIRO
}

(Communicated by Clifford J. Earle, Jr.)

\begin{abstract}
Characterizations are obtained for some classes of Teichmüller-type $L^{\infty}$ functions that are orthogonal to all polynomials in the unit disk.
\end{abstract}

\section{INTRODUCTION}

For $U=\{z:|z|<1\}$ we let

$$
B(U)=\left\{g: g(z) \text { is holomorphic in } U,\|g\|=\iint_{U}|g(z)| d x d y<\infty\right\} .
$$

A function $\nu(z)$ of class $L^{\infty}(U)$ belongs to the annihilating class $N(U)$ if [1]

$$
\iint_{U} \nu(z) g(z) d x d y=0 \text { for all } g \in B(U) \text {. }
$$

We shall be concerned with functions $\nu(z)$ of Teichmüller type; that is, $\nu(z)$ is of the form

$$
\nu(z)=\frac{\overline{\varphi(z)}}{|\varphi(z)|}, \quad z \in U,
$$

where $\varphi(z)$ is single-valued analytic in $U$.

The background of the problems that we deal with is the following question. Let $Q(z)$ be a given quasiconformal self map of $U$. One asks whether there exists a Teichmüller mapping $T(z)$, that is, a quasiconformal mapping of $U$ with complex dilatation of the form

$$
\frac{T_{z}}{T_{z}}=k \frac{\overline{\varphi(z)}}{|\varphi(z)|},
$$

where $k$ is a constant, such that $\left.T\right|_{\partial u}=\left.Q\right|_{\partial U}$ and whether a mapping $T$ of this form is unique. In the simplest special case, $Q(z)=I(z)=z$, we have the trivial solution, $k=0, \varphi$ arbitrary. We are forced to disallow the trivial solution if $T$ is subject to side conditions that dictate that $T$ differs from $I$,

Received by the editors March 10, 1989.

1980 Mathematics Subject Classification (1985 Revision). Primary 30C75; Secondary 30E99.

Key words and phrases. Annihilators, shift theorem, Verschiebungssatz. 
e.g., as in the condition for Teichmüller's Verschiebungssatz, where $T(0)$ is specified distinct from 0 . If $k$ is small, the condition that $\left.T\right|_{\partial U}=\left.I\right|_{\partial U}$ is, up to terms of order $o(k)$, known [1] to be equivalent to (1.1). Thus the problem of finding those $\varphi$ for which a function $\nu$ of Teichmüller type belongs to $N(U)$ provides a first-order model for studying the uniqueness question when the boundary restriction is the identity and $k \neq 0$. Typically, a side condition on $T$ involving a specification of $T$ or a derivative of $T$ at a point of $U$, leads, if $k$ is minimized, to a pole of $\varphi$ at the point. We shall therefore allow $\varphi$ to have poles in $U$. Our principal result (Theorem 3.1) is stated under the assumption that $\varphi(z)$ is meromorphic in $\bar{U}$. It will be clear from the method, however, that some weakening of this assumption would have been possible.

Let $\Omega$ be a bounded region, $\nu \in L^{\infty}(\Omega)$. The integral

$$
u(z)=-\frac{1}{\pi} \iint_{\Omega} \nu(\zeta) \frac{d \xi d \eta}{\zeta-z}, \quad \zeta=\xi+i \eta
$$

is well defined in the complex plane $\mathbf{C}$ and holomorphic in $\mathbf{C} \backslash \bar{\Omega}$. Moreover, as is well known [4], $u(z)$ is uniformly continuous in $\mathbf{C}$, with a modulus of continuity that is $O(\delta \log (1 / \delta))$. Also, $\partial u / \partial \bar{z}=\nu$ in the sense of distributions in $\Omega$. Now if $\Omega$ contains an open disk $V$ in which we have $\nu(z)=\overline{\varphi(z)} /|\varphi(z)|$, where $\varphi(z)$ is holomorphic and nonvanishing in $V$, then $\nu \in C^{\infty}(V)$ and so, by "elliptic regularity theory," $u \in C^{\infty}(V)$. In our case this is also an immediate consequence of the usual Pompeiu representation of $\overline{\Phi(z)} / \Phi^{\prime}(z)$, where $\Phi(z)$ is a single-valued branch of $\int \sqrt{\varphi(z)} d z$ in $V$. For later purposes we shall also require the fact that $\partial u / \partial z$ is locally $L^{2}$ in $\Omega$, an immediate consequence of $(1.3)$ and $\nu \in L^{2}(\Omega)$.

Note that, since the polynomials are dense in $B(U)$ in the $L^{1}$ norm, it suffices that (1.1) hold for $g(z)=z^{n}, n=0,1,2, \ldots$, to imply that $\nu \in$ $N(U)$. Alternatively [1], it is necessary and sufficient that $u(z)$, as defined by (1.3) with $\Omega=U$, vanishes when $|z|=1$.

\section{THE FUNCTIONS $\Lambda(z), \lambda(z)$}

Let $\varphi(z)$ be holomorphic, but not identically vanishing in a region $\Omega$. We define $\Lambda(z), \lambda(z)$ as follows. Let $S_{\varphi}$ be the set of critical points (zeroes or poles) of $\varphi$ in $\Omega$. Starting with a branch of $\Phi(z)=\int \sqrt{\varphi(z)} d z$ in any simply connected subregion of $\Omega \backslash S_{\varphi}, \Phi(z)$ can be continued along any path in $\Omega \backslash S_{\varphi}$. Accordingly, we determine

$$
\Lambda(z)=\overline{\Phi(z)}-\Phi^{\prime}(z) u(z)
$$

along any such path, where $u(z)$ is defined by (1.3).

Since $\Lambda_{\bar{z}}=\overline{\Phi^{\prime}(z)}-\Phi^{\prime}(z)(\overline{\varphi(z)} /|\varphi(z)|)=0$ in $\Omega \backslash S_{\varphi}$ and $\Lambda$ is $C^{\infty}$, it follows that $\Lambda(z)$ is analytic (although not necessarily single-valued) in $\Omega \backslash S_{\varphi}$. We now define

$$
\lambda(z)=\Lambda^{\prime}(z)^{2}, \quad z \in \Omega \backslash S_{\varphi} .
$$


By the order of a critical point we understand an integer $m$, such that $|m|$ equals the order of the zero or pole depending on whether $m$ is respectively positive or negative. If $m=0$, the point is a regular point.

Theorem 2.1. If $\varphi(z)$ is meromorphic in $\Omega$ and has critical set $S_{\varphi}$, then $\lambda(z)$ is also meromorphic in $\Omega$. All poles of $\lambda(z)$ lie in $S_{\varphi}$. If $\varphi(z)$ has a critical point of order $m$ at $z_{0}$ then $\lambda(z)$ has a critical (or regular) point of order $\geq m-2$ at $z_{0}$.

Proof. We note that, locally,

$$
-\Lambda^{\prime}(z)=\frac{\partial}{\partial z}\left(\Phi^{\prime} u\right)=\Phi^{\prime} u_{z}+\Phi^{\prime \prime} u, \quad z \in \Omega \backslash S_{\varphi} .
$$

Continuation of $\Phi^{\prime}(z)$ around a closed curve in $\Omega \backslash S_{\varphi}$ results in multiplication of the function element by \pm 1 , and the same factor acts on $\Phi^{\prime \prime}(z)$. Thus $\lambda(z)$ is single-valued in $\Omega \backslash S_{\varphi}$. In terms of $\varphi(z)$,

$$
\lambda(z)=\varphi(z) u_{z}^{2}+\varphi^{\prime}(z) u(z) u_{z}+\frac{\varphi^{\prime}(z)^{2}}{4 \varphi(z)} u(z)^{2}, \quad z \in \Omega \backslash S_{\varphi} .
$$

Suppose $\varphi(z)$ has a critical point, say at $z=0$. Then $\lambda(z)$ has an isolated singularity at 0 . To investigate the nature of the singularity, suppose first that $\varphi$ has a zero of order $m \geq 1$ at 0 . Then, on some neighborhood $W$ of 0 , we have $\left|\Phi^{\prime}(z)\right| \leq c_{1}|z|^{m / 2}$ and $\left|\Phi^{\prime \prime}(z)\right| \leq c_{2}|z|^{(m / 2)-1}$ (where $c_{j}$ here and in the following denote positive constants), and so, from (2.2) in view of the boundedness of $u$,

$$
|\lambda(z)| \leq c_{3}\left(|z|^{m}\left|u_{z}\right|^{2}+|z|^{m-2}\right), \quad z \in W .
$$

It follows that $\lambda \in L^{1}(W)$, and therefore that $\lambda$ is either regular or has at worst a simple pole at $z=0$. Now, the assertion to be proved is that $\lambda(z)=O\left(|z|^{m-2}\right)$ near 0 . If this is false then, since $\lambda$ is meromorphic at 0 , we must have $|\lambda(z)| \geq c_{4}|z|^{m-3}$ and hence, from (2.3), $\left|u_{z}\right|^{2} \geq c_{5}|z|^{-3}$ for some $c_{5}>0$ contradicting that $u_{z} \in L^{2}(W)$. The proof in the case $\varphi$ has a pole at 0 is nearly identical: if $\varphi$ has a pole of order $|m|$ at 0 then (2.3) still holds, with $m<0$. This readily implies that $|\lambda(z)|^{\varepsilon}$ is integrable on $W$ for some $\varepsilon>0$. From the subharmonicity of $|\lambda|^{\varepsilon}$ on the disk $\left\{z:\left|z-z_{0}\right|<\left|z_{0}\right|\right\}$ with small $\left|z_{0}\right|$ we then get $\left|\lambda\left(z_{0}\right)\right| \leq C\left|z_{0}\right|^{-2 / \varepsilon}$, so again $\lambda$ has at worst a pole at $z_{0}$. The remainder of the proof is nearly identical with that for the case $m>0$ and we omit the details.

Note that Theorem 2.1 allows for the occurrence of a simple pole of $\lambda(z)$ at a simple zero of $\varphi(z)$. As shown in $\S 5$ this possibility may actually take place.

\section{ANNIHILATION WHEN $\varphi$ IS MEROMORPHic in $\bar{U}$}

If $\Lambda(z)$ has the form (2.1) in a half-neighborhood $\omega$ of a boundary point of $U$ and if $\Phi(z)$ is sufficiently nice on a boundary arc and $u(z)$ vanishes 
there, then $\Phi(z)$ can be continued across the arc with the help of (2.1) to $\omega^{*}=\{1 / \bar{z}: z \in \omega\}$. In particular,

Lemma 3.1. Let $\Gamma$ denote an open arc on $\partial U$ all points of which are accessible boundary points of a region $\omega, \omega \subset U$. Suppose $\nu(z)=\overline{\Phi^{\prime}(z)} / \Phi^{\prime}(z), z \in \omega$, where $\Phi(z)$ is holomorphic in $\omega$ and continuous in $\omega \cup \Gamma$. If $\nu \in N(U)$, then $\Phi$ has a holomorphic extension to $\omega \cup \Gamma \cup \omega^{*}$, given by $\Phi(z)=\overline{\Lambda(1 / \bar{z})}, z \in \omega^{*}$.

We can apply this to obtain

Theorem 3.1. Suppose $\varphi(z)$ is meromorphic in the closed unit disk $\bar{U}$. A necessary condition that $\bar{\varphi} /|\varphi|$ be in $N(U)$ is that $\varphi(z)$ be a rational function satisfying the relation

$$
z^{4} \varphi(z)=\overline{\lambda(1 / \bar{z})}, \quad|z|>1 .
$$

Proof. Let $S_{\varphi}$ denote the set of critical points of $\varphi$ in $\bar{U}$. Let $\Gamma$ be an open arc on $\partial U$ containing no points of $S_{\varphi}$, and let $\omega$ be a simply connected subregion of $U$ containing no points of $S_{\varphi}$ such that all points of $\Gamma$ are accessible boundary points of $\omega$. In $\omega$ there exists a single-valued branch of $\Phi(z)$. Let

$$
u(z)=-\frac{1}{\pi} \iint_{U} \frac{\overline{\varphi(\zeta)}}{|\varphi(\zeta)|} \frac{d \xi d \eta}{\zeta-z}, \quad z \in U,
$$

and define $\Lambda(z)$ in $\bar{U} \backslash S_{\varphi}$ by (2.1). $\Lambda(z)$ is also holomorphic in $\omega$, and, by Lemma 3.1, $\Phi$ and $\Lambda$ can be extended to satisfy

$$
\Phi(z)=\overline{\Lambda(1 / \bar{z})}, \quad z \in \omega \cup \Gamma \cup \omega^{*} .
$$

Therefore, $\Phi^{\prime}(z)$ and $\Lambda^{\prime}(z)$ can be extended to satisfy

$$
\Phi^{\prime}(z)=-z^{-2} \overline{\left(\Lambda^{\prime}(1 / \bar{z})\right.}, \quad z \in \omega \cup \Gamma \cup \omega^{*} .
$$

Squaring, we obtain (3.1) and conclude that $\varphi$ is rational. This completes the proof.

\section{4. $\sqrt{\varphi(z)}$ HOLOMORPHIC}

In the special case when $\varphi(z)$ has a single-value square root $f(z)$ which is holomorphic in $U$ and meromorphic in $\bar{U}$, Theorem 3.1 can be replaced by a more explicit condition, as follows.

Theorem 4.1. Suppose $f(z)$ is a function, not identically 0 , holomorphic in $U$ and meromorphic in $\bar{U}$. A necessary and sufficient condition that $\bar{f} / f \in N(U)$ is that $f(z)=F^{\prime}(z), z \in U$, where $F(z)$ is a nonconstant rational function with the following properties:

(a) All poles of $F(z)$ are on $\partial U$; that is, $F$ is holomorphic for $|z|<1$ and for $|z|>1$, including at $\infty$.

(b) If $f\left(z_{0}\right)=0, f^{\prime}\left(z_{0}\right)=0, \ldots, f^{(s)}\left(z_{0}\right)=0$, for some $z_{0} \in U$ and $s \geq 0$, then $F(z)-F\left(z_{0}\right)$ has a zero of multiplicity at least $s+1$ at $z=1 / \bar{z}_{0}$. 
Proof. In terms of the notation of the previous sections, we have $\bar{\varphi} /|\varphi|=$ $\bar{\Phi}^{\prime} / \Phi^{\prime}$; let $f(z)=\Phi^{\prime}(z), F(z)=\Phi(z)$, where $F(z)$ is holomorphic in $U$. We apply Lemma 3.1 with $\omega=U$, where $\Gamma$ is any open arc on $\partial U$ containing no poles of $f$. By Lemma 3.1, $F(z)$ extends as a rational function to $\widehat{\mathbf{C}}$, satisfying

$$
F(z)=\overline{\Lambda(1 / \bar{z})}, \quad 1<|z| \leq \infty .
$$

The remainder of the assertion follows from Theorem 5 of [3].

An implicit consequence of Theorem 4.1 is that $f$ cannot have any first-order poles on $\partial U$ if $\bar{f} / f$ belongs to $N(U)$. Following similar results of Ortel [2], this fact can be formulated quantitatively as follows:

Theorem 4.2. Suppose $f(z)$ is measurable in $U$ and, for some $z_{0} \in \partial U$ and $c \neq 0$,

$$
\lim _{z \rightarrow z_{0}}\left(z-z_{0}\right) f(z)=c .
$$

If $\|\nu\|_{\infty} \leq 1$, and if $\nu(z)=\overline{f(z)} / f(z)$ in a neighborhood of $z_{0}$ in $U$, then

$$
\sup \left\{\left|\iint_{U} \nu(z) g(z) d x d y\right| /\|g\|: g \in B(U)\right\}=1 .
$$

Proof. It suffices to show that if $\nu(z) \in L^{\infty}(U)$, and if, for some $z_{0} \in \partial U$,

$$
\lim _{z \rightarrow z_{0}}\left(\overline{z-z_{0}} / z-z_{0}\right) \nu(z)=1
$$

then

$$
\sup \left\{\left|\iint_{U} \nu(z) g(z) d x d y\right| /\|g\|: g \in B(U)\right\} \geq 1 .
$$

If we map $U$ conformally onto the right-half plane $H$, transforming $\nu$ and $g$ so that $\nu(z) \overline{d z} / d z$ and $g(z) d z^{2}$ remain, respectively, invariant, we can replace $U$ by $H$ and $z_{0}$ by 0 in (4.1) and (4.2). In $H$, consider

$$
g_{n}(z)=\frac{z^{1 / n}}{\pi n(z+1) z^{2}}, \quad n=2,3, \ldots,
$$

where $z^{1 / n}$ denotes the principal branch. Since $\max (1,|z|) \leq|z+1| \leq \rho+1$ when $z \in H,|z| \leq \rho$, we have

$$
\begin{gathered}
\frac{\rho^{1 / n}}{1+\rho} \leq \iint_{\{|z|<\rho\} \cap H}\left|g_{n}(z)\right| d x d y \leq \rho^{1 / n}, \\
\iint_{\{|z|>\rho\} \cap H}\left|g_{n}(z)\right| d x d y \leq \frac{1}{(n-1) \rho^{1-1 / n}}, \quad n=2,3, \ldots
\end{gathered}
$$

It follows that

$$
\lim _{n \rightarrow \infty} \iint_{H}\left|g_{n}(z)\right| d x d y=1 .
$$


Near $z=0$, when $n$ is large, $n \pi g_{n}(z)$ behaves approximately like $z^{-2}$ in $H$, while, by (4.1), given $\varepsilon>0$,

$$
|\nu(z)-(z / \bar{z})|<\varepsilon \quad \text { for }|z|<\delta, z \in H,
$$

if $\delta>0$ is sufficiently small. As a consequence,

$$
\lim _{n \rightarrow \infty}\left|\iint_{H} \nu(z) g_{n}(z) d x d y\right|=1 \text {, }
$$

completing the proof.

Remark. In view of the Hahn-Banach Theorem, we have an equivalent dual form of Theorem 4.2; namely,

The distance in $L^{\infty}(U)$ from $\nu$ to $N(U)$ is not less than 1 .

\section{EXAMPLES}

Example 5.1. $\varphi(z)=z(1-a z)^{2}, z \in U$, where $|a|<1$. According to Theorem 2.1 , we can expect $\lambda(z)$ to have at worst a pole of order one at $z=0$. As the result of a computation we obtain

$$
u(z)=\frac{2}{1-a z}\left[\frac{|z|^{3}}{3 z^{2}}-\frac{a^{2}}{3}-\frac{\bar{a}|z|^{5}}{5 z^{3}}+\frac{a^{2}|a|^{2}}{5}\right], \quad|z|<1,
$$

and, by (2.1),

$$
\lambda(z)=\left(\frac{1}{3}-\frac{|a|^{2}}{5}\right)^{2} \frac{a^{4}}{z}, \quad|z|<1 .
$$

Thus, $\lambda(z)$ has a simple pole when $a \neq 0$, but when $a=0, \lambda(z)$ is identically zero.

Example 5.2. $\lambda(z)=\frac{1}{z},|z|<1$. We have

$$
\frac{\overline{\varphi(z)}}{|\varphi(z)|}=\frac{z}{|z|}=e^{i \theta}, \quad z=r e^{i \theta}, 0 \leq r<1 \text {. }
$$

Therefore

$$
\frac{\overline{\varphi(z)}}{|\varphi(z)|} \in N(U)
$$

So, we can use this to illustrate Theorem 3.1. A computation gives

$$
u(z)= \begin{cases}-2(1-|z|), & |z|<1, \\ 0, & |z|>1\end{cases}
$$

and by (2.1) we obtain $\lambda(z)=z^{-3}, z \in U$. Condition (3.1) is evidently satisfied.

Example 5.3 [3]. $F(z)$ schlicht in $U, F(z)$ rational, $F(U)=$ unbounded region. Concrete examples are obtained when $F(U)$ is a halfplane, or a plane slit along a radial line, or the "exterior" of a parabolic region. 
In all cases the hypotheses of Theorem 4.1 are satisfied, with condition (b) satisfied vacuously, since $F^{\prime}(z)$ does not vanish in $U$.

Example 5.4. The purpose of this example is to show that hypothesis (b) of Theorem 4.1 may hold nonvacuously. To construct an example we replace $U=\{|z|<1\}$ by $H_{1}=\{\operatorname{Im} w>0\}$. If $w(z)$ is a conformal map of $U$ onto $H_{1}$ and if we define $\widetilde{F}(w)=F(z)$, then $\overline{\widetilde{F}^{\prime}(w)} / \widetilde{F}^{\prime}(w)$ will belong to $N\left(H_{1}\right)$ if and only if $\overline{f(z)} / f(z)$ belongs to $N(U)$. Conditions (a) and (b) become

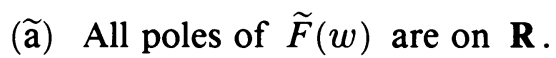

$(\widetilde{\mathbf{b}})$ If $\widetilde{F}^{\prime}\left(w_{0}\right)=\widetilde{F}^{\prime \prime}\left(w_{0}\right)=\cdots=\widetilde{F}^{(s+1)}\left(w_{0}\right)=0$ for some $w_{0} \in H_{1}, s \geq 0$, then $\widetilde{F}(w)-\widetilde{F}\left(w_{0}\right)$ has a zero of multiplicity at least $s+1$ at $w=\bar{w}_{0}$.

We define

$$
\widetilde{F}(w)=\frac{1}{w}-\frac{1+i}{w-1}-\frac{1-i}{w+1},
$$

obtaining

$$
\begin{aligned}
w^{2}(w-1)^{2}(w+1)^{2} \widetilde{F}^{\prime}(w) & =w^{4}+4 i w^{3}+4 w^{2}-1 \\
& =(\alpha-1)\left(\alpha^{3}+5 \alpha^{2}+\alpha+1\right),
\end{aligned}
$$

where $w=i \alpha$. Since $\alpha^{3}+5 \alpha^{2}+\alpha+1$ has no roots with $\operatorname{Re} \alpha>0, \widetilde{F}^{\prime}\left(w_{0}\right)=0$ in $H_{1}$ if and only if $w_{0}=i$; moreover, $\widetilde{\sim}^{\prime \prime}(i) \neq 0$. Since $\widetilde{F}(-i)=\widetilde{F}(i)$, both $(\widetilde{\mathbf{a}})$ and $(\widetilde{\mathbf{b}})$ are satisfied.

\section{REFERENCES}

1. Lars V. Ahlfors, Some remarks on Teichmüller's space of Riemann surfaces, Ann. of Math. 74 (1961), 171-191.

2. Marvin Ortel, Extremal quasiconformal mappings with angular complex dilatation, Indiana Univ. Math. J. 31 (1982), 435-447.

3. Edgar Reich and Kurt Strebel, On quasiconformal mappings which keep the boundary fixed, Trans. Amer. Math. Soc. 138 (1969), 211-222.

4. I. N. Vekua, Generalized analytic functions, Pergamon Press, Oxford, 1962.

School of Mathematics, Vincent Hall, University of Minnesota, Minneapolis, MinNESOTA 55455

Matematiska Institutionen, Kungl. Tekniska Högskolan, S-10044 Stockholm, Sweden 